\title{
EL DISEÑO UNIVERSAL COMO MEDIO PARA ATENDER A LA DIVERSIDAD EN LA EDUCACIÓN. UNA REVISIÓN DE CASOS DE ÉXITO EN LA UNIVERSIDAD
}

\author{
Sergio Sánchez Fuentes \\ Universidad de Burgos \\ Emiliano Díez Villoria \\ Universidad de Salamanca \\ Rosario Ángela Martín Almaraz \\ Universidad Rey Juan Carlos
}

\begin{abstract}
RESUMEN: Este trabajo tiene como objetivo principal describir una serie de casos de éxito en la aplicación del paradigma del diseño universal a la educación universitaria como medio para atender la diversidad en las aulas. Para ello, en primer lugar, se presentan los principales enfoques relacionados con aplicación del diseño universal en ámbitos educativos; y, en segundo lugar, se realiza una breve revisión de las principales investigaciones que avalan la aplicación del diseño universal a entornos educativos, organizando la revisión en torno a tres grandes categorías: (a) estudios sobre formación de docentes; (b) estudios sobre implantación de currículos basados en diseño universal; y (c) estudios que analizan las percepciones de los estudiantes. Se concluye que, atendiendo a los resultados de los trabajos revisados, el diseño universal es una metodología válida para atender a los estudiantes universitarios con capacidades diversas.
\end{abstract}

PALABRAS CLAVE: Diseño universal, diversidad, universidad, discapacidad, inclusión.

\section{UNIVERSAL DESIGN SUCESSFUL AS A METHOD OF DEALING DIVERSITY IN EDUCATION. A REVIEW OF SUCCESS CASES ON UNIVERSITY}

\footnotetext{
ABSTRACT: The main objective of this paper is to describe a variety of successful cases related to the implementation of universal design as a mean to address diversity in postsecondary educational settings. First, main approaches to implementation of universal design in educational settings are presented;
} 
and second, a brief review of the main studies that support the application of universal design to learning environments is done, organizing the review around three broad categories: (a) studies on teacher training about universal design for learning; (b) studies on introduction of curricula based on universal design; and (c) studies examining student's perceptions after universal design implementation. It is concluded that, based on the results of the studies reviewed, universal design is a valid methodology to address diversity of university students.

KEYWORDS: Universal design, diversity, university, disability, inclusion.

Recibido: 16/04/2015

Aceptado. 24/07/2015

Correspondencia: Sergio Sánchez, Facultad de Educación, Departamento de Ciencias de la Educación, Universidad de Burgos, C/Villadiego s/n, 09001 Burgos. Email: ssfuentes@ubu.es.

\section{INTRODUCCIÓN}

La Organización de las Naciones Unidas para la Educación, la Ciencia y la Cultura determinó que la educación inclusiva no es una cuestión marginal, sino que es crucial para lograr una educación de calidad para todos los estudiantes (UNESCO, 2008). En la actualidad, el concepto de inclusión está presente en la educación obligatoria debido al esfuerzo realizado en las últimas décadas en España (CIDE, 2008); sin embargo, su presencia en las etapas universitarias es mucho más difusa. Así, David (2004) señala que la inclusión en la educación superior es un término con una gran vigencia internacional, pero cómo se conceptualiza y determina es un tema mucho más complejo.

$\mathrm{Y}$ es que, como señalan diferentes autores, a nivel mundial existen alrededor de un $6 \%$ de estudiantes con algún tipo de discapacidad (Horn y Nevill, 2006; Lewis y Farris, 1999). Concretamente en Europa, se estima que hay unos 84 millones de estudiantes en las aulas, y de ellos, aproximadamente el 20\% requerirá alguna actuación relacionada con la atención a la diversidad a lo largo de su trayectoria académica (EURYDICE, 2000). Dependiendo de cómo se diagnostique, cómo se consideren las necesidades educativas especiales, o cómo se traten en el ámbito educativo, los estudiantes con discapacidad pueden representar entre el $2 \%$ y el $18 \%$ de la población en edad escolar (EADSNE, 2006).

Por lo tanto, ante esta situación, la comunidad universitaria se debe plantear la búsqueda de métodos y enfoques que favorezcan esa inclusión educativa en la etapa universitaria. Y en este contexto, el diseño universal se presenta como uno de los enfoques clave en relación a los objetivos de inclusión, y más en concreto, respecto al incremento en la participación de la personas con discapacidad en la universidad (Sánchez, Díez, Verdugo, Iglesias y Calvo, 2011).

El diseño universal fue descrito por Mace (1985), como el diseño de productos y entornos de fácil uso para el mayor número de personas posible, sin la necesidad de 
adaptarlos o rediseñarlos de una forma especial. Sin embargo, esta concepción es aplicable a ámbitos diferentes a la educación, pero yendo un paso más adelante y, como bien señalan McGuire, Scott y Shaw: "el diseño universal aplicado a la educación puede ser un nuevo paradigma que permita hacer efectiva la implementación de la inclusión y proporcionar acceso a la educación general del currículo" (2006: 167).

\section{El Diseño Universal del Aprendizaje en la educación superior}

La necesidad de disponer de currículos accesibles, que contemplen la diversidad de estudiantes dentro de las aulas, debería ser un aspecto prioritario a tener en cuenta en los procesos de diseño curricular (Díez et al., 2011). Más específicamente, incluso la propia legislación de las enseñanzas universitarias hace mención a dicha necesidad (Sánchez, 2015).

No existe un único enfoque que avale los beneficios de aplicar el enfoque del diseño universal al diseño curricular de las actuaciones pedagógicas (Sala, Sánchez, Giné, Díez, 2014); más bien, en los último años, han ido surgiendo diversas maneras para integrar el diseño universal en la universidad.

Probablemente, uno de los principales enfoques, es el denominado diseño universal para el aprendizaje (DUA). El Center for Applied Special Technology (CAST) define el DUA como un marco científicamente válido para guiar la práctica educativa, que proporciona flexibilidad en las formas de presentar la información, en los modos en los que los estudiantes responden o demuestran sus conocimientos y habilidades, y en la manera en que los estudiantes participan y se implican en su propio aprendizaje (CAST, 2011). Además, el DUA pretende reducir las barreras en la enseñanza, proporcionando adaptaciones, apoyos y retos apropiados, y manteniendo unas altas expectativas de logro para todos los estudiantes. Los tres principios del DUA aparecen descritos en la siguiente tabla.

Tabla 1. Principios y Pautas del Diseño Universal para el Aprendizaje (Adaptado de NCUDL, 2012)

\begin{tabular}{|c|c|c|c|}
\hline & $\begin{array}{l}\text { Principio I. Proporcionar } \\
\text { múltiples formas de } \\
\text { representación (el "qué" } \\
\text { del aprendizaje) }\end{array}$ & $\begin{array}{l}\text { Principio II. Proporcionar } \\
\text { múltiples formas para la } \\
\text { acción y la expresión (el } \\
\text { "cómo" del aprendizaje) }\end{array}$ & $\begin{array}{c}\text { Principio III. Proporcionar } \\
\text { múltiples formas de } \\
\text { participación* (el "por } \\
\text { qué" del aprendizaje) }\end{array}$ \\
\hline \multirow{3}{*}{$\frac{5}{5}$} & $\begin{array}{l}\text { 1. Proporcionar opciones } \\
\text { para la percepción }\end{array}$ & $\begin{array}{l}\text { 4. Proporcionar opciones } \\
\text { para la interacción física }\end{array}$ & $\begin{array}{l}\text { 7. Proporcionar opciones } \\
\text { para captar el interés }\end{array}$ \\
\hline & $\begin{array}{l}\text { 2. Proporcionar múltiples } \\
\text { opciones para el lenguaje, } \\
\text { los símbolos y las expre- } \\
\text { siones matemáticas }\end{array}$ & $\begin{array}{l}\text { 5. Proporcionar opciones } \\
\text { para la expresión y la co- } \\
\text { municación }\end{array}$ & $\begin{array}{l}\text { 8. Proporcionar opciones } \\
\text { para mantener el esfuerzo } \\
\text { y la persistencia }\end{array}$ \\
\hline & $\begin{array}{l}\text { 3. Proporcionar opciones } \\
\text { para la comprensión }\end{array}$ & $\begin{array}{l}\text { 6. Proporcionar opciones } \\
\text { para las funciones ejecu- } \\
\text { tivas }\end{array}$ & $\begin{array}{l}\text { 9. Proporcionar opciones } \\
\text { para la auto-regulación }\end{array}$ \\
\hline
\end{tabular}


Junto al DUA, también han ido surgiendo otros enfoques basados en el diseño universal. Por ejemplo, otro paradigma similar es el que Bryson (2003) define como diseño universal instruccional aplicado a la educación. Desde este enfoque se plantea el incremento de la "capacidad instruccional" de los contextos educativos de personas adultas prestando atención al diseño, la ejecución y la evaluación del desempeño del estudiante y de los contenidos (Bryson, 2003).

Roberts, Park, Brown y Cook (2011) proponen el denominado diseño universal para la instrucción. Como bien señala Pliner y Johnson (2004), el diseño universal para la instrucción pretende expandir las metodologías instruccionales para que todos los estudiantes con discapacidad, y todos los estudiantes con necesidades diversas de aprendizaje, logren un acceso igualitario al proceso de enseñanza y aprendizaje.

Finalmente, tenemos el enfoque denominado diseño universal en educación (Burgstahler, 2002); aunque, como señalan Ruiz, Solé, Echeita, Sala y Datsira (2012), no se puede considerar como un paradigma en sí mismo, ni de la misma envergadura que los otros enfoques que se acaban de describir. La diferencia fundamental que se puede encontrar es la utilización de la palabra educación, que conllevaría una concepción más amplia que aprendizaje, en lo que a variedad de procesos y situaciones se refiere.

Una vez presentados algunos de los principales enfoques que tienen el diseño universal como base para atender a la diversidad en la universidad, este trabajo se plantea como objetivo, describir una serie de casos de éxito donde se ha aplicado el diseño universal en espacios pedagógicos.

\section{BUENAS PRÁCTICAS EN LA UNIVERSIDAD BASADAS EN EL DISEÑO UNIVERSAL PARA}

\section{ATENDER A LA DIVERSIDAD}

En este apartado se van a presentar algunos de los principales trabajos que avalan la aplicación del diseño universal en la universidad. La descripción de dichos casos de éxito se va a realizar en base a diferentes estrategias a la hora de aplicar el diseño universal en la universidad. De esta manera, y en primer lugar, se describen los casos que tiene relación con la formación que los profesores universitarios tienen sobre cómo atender a la diversidad y su relación con el diseño universal. Así, diferentes estudios han demostrado que el entrenamiento eficaz de profesores (tanto de enseñanzas básicas, como de enseñanzas superiores), es beneficioso para la creación de un currículum integrador para los estudiantes (Engleman y Schmidt, 2007; Spooner, Baker, Harris, Ahlgrim-Delzell y Browder, 2007).

Un buen ejemplo es el estudio realizado por Spooner et al. (2007), en el que se demuestra que incluso acciones formativas breves dirigidas a la formación del profesorado sobre cómo desarrollar un currículo basado en DUA favorecen el desarrollo de estrategias inclusivas en la creación de contenidos de aprendizaje. Este estudio consistió en la realización de un seminario de una única hora de duración presentando los principios del DUA y unas pautas básicas de cómo implementarlo en el currículo. Los análisis demostraron que los grupos donde se había realizado la formación sobre DUA fueron capaces de crear lecciones más inclusivas que las realizadas por un grupo control. 
A través del denominado "Proyecto ACCESS", en la Universidad de Colorado, se Ilevó a cabo un estudio (Schelly, Davies y Spooner, 2011) donde se describía cómo, a través de la formación a docentes, se lograban mejoras en el aprendizaje, y cómo éstas eran percibidas por los estudiantes. Los autores utilizaron diferentes metodologías en este estudio. En primer lugar, el estudio comenzaba con la creación de un grupo focal compuesto por los profesores que impartían la asignatura de "Introducción a la Psicología". En dicho grupo se discutieron los principios del DUA y cómo éstos podrían ayudar a la impartición de su asignatura. Durante el semestre en el que se impartía la asignatura, los profesores realizaron reuniones semanales de una hora de duración, donde el foco principal de estudio era la aplicación de medidas basadas en el paradigma del DUA. La investigación exigía que el currículum de la asignatura fuera modificado siguiendo las pautas marcadas en la formación recibida por parte de los profesores. Adicionalmente, los profesores fueron instruidos sobre el modo adecuado para crear documentos Word, presentaciones PowerPoint, documentos PDF y páginas Web con HTML siguiendo los estándares de accesibilidad de contenidos digitales. Además, los investigadores desarrollaron un cuestionario, basado en los tres principios del DUA, con un total de 24 preguntas relacionadas con la aplicación de los principios en la asignatura. Inicialmente este cuestionario fue suministrado a un total de 1.362 estudiantes que previamente estaban matriculados en la asignatura de aquellos profesores que habían participado en el grupo focal.

Los resultados mostraron un incremento significativo en la percepción de los estudiantes sobre la utilización de estrategias basadas en los principios de diseño universal en 14 de los 24 ítems del cuestionario. Los autores del trabajo concluyeron que el entrenamiento de los profesionales, siguiendo las estrategias de implementación del DUA, es percibido por los estudiantes como algo positivo y que favorece la educación en las aulas universitarias para todos los estudiantes, al margen de sus características individuales.

Otro ejemplo, esta vez basado en el entrenamiento de docentes universitarios en la creación de cursos de formación on-line siguiendo los principios del DUA, es el presentado por Engleman y Schmidt (2007). Este estudio, realizado con 216 participantes, demostró que los estudiantes prefieren los cursos desarrollados bajos los principios del DUA. Esta afirmación se basa, fundamentalmente, en la variedad de opciones que se derivan de la aplicación de diseño universal y la posibilidad de adecuarlas al propio estilo de aprendizaje. Los estudiantes se encontraron más cómodos en un ambiente educativo cuando la programación fue secuenciada, estructurada y definida desde el comienzo siguiendo los parámetros de la accesibilidad. Además, la presentación del material de una manera más visual favoreció el aprendizaje de los contenidos.

Otros trabajos de investigación se han centrado, más específicamente, en la implantación de currículos basados en diseño universal y la verificación de su utilidad.

Rose, Harbour, Johnston, Daley y Abarbanell (2006), describieron una experiencia sobre cómo se implementó el curso denominado "El Reto de las Diferencias Individuales" en la Universidad de Harvard (Estados Unidos) siguiendo los principios del DUA. Como característica fundamental de la realización de este seminario habría que destacar la cantidad de recursos que se pusieron a disposición de los estudian- 
tes que formaban parte del mismo. Entre los recursos más interesantes estaban, por ejemplo: (a) un intérprete de lengua de signos para aquellos estudiantes sordos; (b) texto alternativo en cualquier imagen presentada a lo largo de la duración del curso para personas con baja visión; y (c) grabación en vídeo de las lecciones magistrales y su posterior alojamiento en un página Web creada específicamente para el desarrollo del curso -lo que permite visualizar cualquier parte de las conferencia en el momento deseado después de la lección presencial-. Como conclusión de este trabajo los autores determinaron que parte de las dificultades de aprendizaje están ubicadas en el ambiente y no tanto en los estudiantes (Rose et al., 2006). También concluyeron que el DUA puede beneficiar a los estudiantes con algún tipo de discapacidad, aunque, en general, la adopción de estrategias basadas en este enfoque favorece a todos los estudiantes.

Meo (2008) también presentó otro interesante trabajo de aplicación de los principios del DUA en el contexto de un programa de mejora de la comprensión lectora. Además, en este trabajo se propone el proceso PAL (Planing for All Learners), que proporciona a los docentes una serie de pasos que se pueden utilizar en la planificación de currículos dirigidos a mejorar resultados de todos los estudiantes.

En lo que se refiere a la implementación de currículos en la educación universitaria, una de las principales experiencias ha sido la llevada a cabo por la Universidad de Guelph. En el contexto de una investigación denominada "Proyecto de Diseño Universal Instruccional", Yuval, Procter, Korabik y Palmer(2004) iniciaron la puesta en marcha de una serie de acciones en nueve cursos impartidos en dicha universidad, que fueron modificados siguiendo los principios del diseño universal instruccional. Los objetivos principales del programa fueron dos, fundamentalmente: (a) estimular a los estudiantes en la aplicación de los principios del diseño universal aplicado a la educación en los cursos; y (b) evaluar el impacto de la aplicación de estos principios (McGuire et al., 2006).

Los cursos seleccionados en este proyecto fueron rediseñados bajo la óptica del diseño universal instruccional. Para ello, en algunos cursos se modificó por completo el currículo, y en otros, se mejoró el ya existente. La evaluación incluía cuestionarios a los estudiantes, observaciones en clase, y entrevistas, tanto a profesores como a los propios alumnos. El objetivo fundamental de dicha evaluación era analizar el impacto de las medidas de diseño universal en los estudiantes. Así, a los estudiantes que participaron en el estudio, se les administraron cuestionarios en tres fases diferentes del proyecto: al comienzo, hacia la mitad del curso y al finalizar. Los resultados mostraron una correlación entre el nivel de implementación de medidas de diseño universal y la sensación de auto-eficacia, además de verificarse más emociones positivas por parte de los estudiantes. Las entrevistas a los estudiantes se realizaron en dos ocasiones, una al comienzo del curso y la siguiente pasados tres meses desde el primer encuentro. Como método de entrevista se utilizó un formato de grupos focales. Estas entrevistas revelaron aspectos específicos que se deberían mejorar antes de comenzar el curso. Finalmente, un punto a destacar, fue la evaluación proporcionada por los propios profesores que fueron entrevistados en dos ocasiones. En ellas, los profesores manifestaron un incremento en los conocimientos acerca del DUI, así como un mayor compromiso con este paradigma para sus futuras actividades académicas. 
Para finalizar, y siguiendo con la estructura planteada al comienzo del artículo, se describen aquellos trabajos que se basan en el análisis de las percepciones de los principales implicados, es decir, los propios estudiantes con capacidades diversas. Y es que varios estudios han presentado reflexiones y respuestas de los estudiantes con discapacidad en las aulas universitarias en relación al diseño universal.

Uno ejemplo es el capítulo de Durre, Richardson, Smith, Shulman y Steele (2008) titulado Universal Design for Instruction. Reflections of Students en el libro "Universal Design in Higher Education" (Burgstahler y Cory, 2008). En dicho capítulo los autores muestran las experiencias y puntos de vista de varios estudiantes respecto a la aplicación de los principios del diseño universal. Las áreas que tuvieron en cuenta para categorizar las respuestas de los estudiantes fueron: (a) el clima de clase; (b) la interacción; (c) los espacios físicos y los productos; (d) métodos de implementar enseñanza; (e) los recursos de tecnologías e información; (f) el feedback; (g) la evaluación; y (h) las adaptaciones. En resumen, el trabajo concluye planteando que "las estrategias del diseño universal representan una manera de enseñar que minimizan las necesidades de adaptaciones específicas" (Durre et al., 2008: 95). Es más, incluso, los estudiantes con discapacidades invisibles han descrito el enfoque del diseño universal como un marco que proporciona nuevas oportunidades en el proceso de enseñanza-aprendizaje, no solo para los estudiantes con alguna discapacidad, sino para todos los estudiantes diversos que caracterizan las aulas del siglo XXI (Spencer y Romero, 2008).

En otro estudio Ilevado a cabo por Arter, Perlis, Ruthkosky, Burkhouse y Holmes (2008), se demostró que la participación y la motivación por parte de los estudiantes aumentaba en más del $50 \%$ cuando la tecnología se utiliza adecuadamente y siguiendo los principios del DUA. Así, en estudios sobre educación basada en el uso de la tecnología se ha verificado en los últimos años una mayor presencia de estudiantes con discapacidad en los cursos on-line ofertados por las universidades (Cook y Gladhart, 2002).

En España, una de las principales investigaciones centradas en el análisis de las percepciones de los estudiantes con discapacidad fue el estudio llevado a cabo por Castellana y Sala (2005). En este trabajo se examinaron las necesidades y dificultades que encontraban los estudiantes universitarios con discapacidad física y sensorial dentro del aula, y las necesidades y dificultades que el profesor universitario tenía para atender a dichos estudiantes. Con una metodología basada en entrevistas semiestructuradas, las autoras hicieron un análisis de las respuestas de estos colectivos sobre tres asuntos: (a) la formación en el ámbito de la discapacidad por parte de los profesores; (b) la creación de servicios especializados en la atención a estudiantes con discapacidad; y (c) la situación de los profesores y los estudiantes en relación a la discapacidad. Como resultados fundamentales que pueden resumir las necesidades expresadas en los tres apartados, los participantes destacaron la necesidad de formar al profesorado en temas de discapacidad y de nuevas metodologías pedagógicas adecuadas ante la diversidad del aula, desarrollar actitudes positivas hacia las personas con discapacidad, disponer de los materiales con antelación y que éstos sean accesibles.

Finalmente, y para concluir con la descripción de casos de éxito sobre la aplicación del paradigma del diseño universal en la educación universitaria, es necesario 
destacar la importancia que este enfoque está adquiriendo en la educación superior. En un reciente trabajo publicado por Roberts et al. (2011) se realizó una revisión sistemática dirigida a identificar los principales estudios que han descrito aplicaciones de diseño universal en la educación post-secundaria. En la revisión se incluyeron estudios empíricos, cuantitativos, cualitativos y mixtos relacionados con la creación y aplicación de currículo basado en los principios de accesibilidad y diseño universal en la educación, y que estuvieran indexados en las principales bases de datos como ERIC, PsychInfo, Academic Search Premier y Social Sciences Citation Index. Los autores concluyen su revisión planteando que, aunque la literatura apoya distintas formas de promoción del diseño universal en educación -similares a las revisadas en este mismo trabajo-, todavía se constata una carencia de investigaciones que muestren la efectividad del diseño universal como medio para mejorar resultados concretos de estudiantes de post-secundaria (e.g., ratios de graduación). Además, la alta frecuencia de estudios que utilizan metodologías cualitativas limita la generalización de los principales hallazgos.

\section{Conclusiones}

Desde los inicios de lo que hoy denominamos educación inclusiva o escuelas inclusivas (Ainscow, 2006; Booth y Ainscow, 2002), uno de los objetivos más intensamente perseguidos por parte de la comunidad educativa ha sido la consecución de la plena participación de la diversidad de alumnado que caracteriza las aulas de los distintos niveles educativos. Gracias a los avances legislativos de las últimas décadas, la mejora de los derechos de los estudiantes que tienen algún tipo de dificultad se han constituido como un importante logro de cara a facilitar la plena participación de cualquier estudiante en la vida académica (Martínez, De Haro y Escarbajal, 2010).

En este trabajo se han presentado diferentes enfoques con un único objetivo común: crear currículos que faciliten las mismas oportunidades para todos los estudiantes. Como bien han señalado diversos autores (Bryson, 2003; Burgstahler, 2002; Pliner y Johnson, 2004; Rose y Meyer, 2002), la aplicación del diseño universal al ámbito educativo promueve la creación de currículos flexibles que se pueden adaptar a las necesidades educativas de cada estudiante. Diseño universal para el aprendizaje, diseño universal instruccional, diseño universal para la instrucción o diseño universal en educación son los principales enfoques que han ido surgiendo a lo largo de las dos últimas décadas en un intento de describir como el paradigma del diseño para todos es aplicable al ámbito educativo.

De manera global, las principales investigaciones que se han identificado en este trabajo han mostrado como la aplicación del enfoque del diseño universal en el proceso educativo, en general, y en el ámbito universitario, en particular, benefician tanto a estudiantes como a profesores y, por tanto, se configura como un paradigma válido cuando el objetivo es mejorar las condiciones de enseñanza y aprendizaje de todas las personas.

En definitiva, desde la aparición de la educación inclusiva como marco general en el que se pretende que la educación sea lo más accesible para todos los estudiantes, se ha realizado un especial esfuerzo para conseguir que las personas diversas, 
incluidas las personas con discapacidad, dispongan de las mismas oportunidades en su educación. Y un medio para lograr este objetivo es, como bien señalan Verdugo y Rodríguez-Aguilella (2012), agilizar la puesta en marcha de iniciativas centradas de educación inclusiva atendiendo a la filosofía del diseño universal.

Independientemente de que se trate de diseño universal para el aprendizaje, diseño universal para la instrucción, diseño universal instruccional o diseño universal en educación, este trabajo demuestra que en la comunidad universitaria se están implantando medidas basadas en el diseño universal, y que éstas, resultan beneficiosas para los estudiantes con discapacidad, pero también, para aquellos estudiantes que no presentan, a priori, ninguna dificultad en su proceso de enseñanza y aprendizaje. Es por tanto, tarea de las administraciones educativas, de las universidades, y de los propios profesores, re-prensar sus prácticas docentes desde un paradigma proactivo como es el del diseño universal aplicado a la educación superior.

\section{REFERENCIAS BIBLIOGRÁFICAS}

Ainscow, M. (2006). From special education to effective schools for all: a review of progress so far. En L. Floiran (ed.), The Sage Handbook on Special Education (pp. 146-159). London: Sage.

Arter, P., Perlis, S., Ruthkosky, K., Burkhouse, E., y Holmes, J. (2008). Using Student Response Systems for Curricular Redesign in Education Courses. En Proceedings of Society for Information Technology y Teacher Education International Conference 2008 (pp. 5203-5208). Chesapeake, VA. Recuperado de: http://www.editlib. org/p/28102.

Booth, T., y Ainscow, M. (2002). Index for Inclusion. Developing learning and participation in schools. CSIE Mark Vaughan.

Bryson, J. (2003). Universal instructional design in postsecondary setting. Recuperado de: www.mohawkcollege.ca/Assets/StudentDev/.../UID+Manual.pdf.

Burgstahler, S. (2002). Universal Design of distance Learning. Information Technology and Disabilities Journal, 13(1). Recuperado de: http://easi.cc/itd/volume8/ number1/burgstah.html.

Burgstahler, S., y Cory, R.C. (Eds.). (2008). Universal Design in Higher Education: From Principles to Practice. Cambridge, MA.: Harvard Education Press.

Castellana, M., y Sala, I. (2005). La universidad ante la diversidad en el aula. Aula Abierta, 85, 57-84.

Center for Applied Special Technology (2011). Universal Design for Learning Guidelines version 2.0. Wakefield, MA. Recuperado de http://www.udlcenter.org/sites/ udlcenter.org/files/UDL_Guidelines_Version_2.0_(Final)_3.doc.

Centro de Investigación y Documentación Educativa (2008). El Desarrollo de la Educación en España. Informe Nacional de España. Madrid: Ministerio de Educación, Política Social y Deporte.

Cook, R., y Gladhart, M. (2002). A survey of online issues and instructional strategies for postsecondary students with learning disabilities. Information Technology and Disabilities Journal, 13(1). Recuperado de: http://easi.cc/itd/volume8/number1/ gladhart.html. 
David, M.E. (2004). Equality and Equity in Higher Education: Learning to develop new paradigms from the US experience. European Educational Research Journal, 3(4), 813-819.

Díez, E., Alonso, A., Verdugo, M.A., Campo, I., Sancho, I., Sánchez, S., y Moral, E. (2011). Espacio Europeo de Educación Superior: estándares e indicadores de buenas prácticas para la atención a estudiantes universitarios con discapacidad. Salamanca: Instituto de Integración en la Comunidad, Universidad de Salamanca.

Durre, I., Richardson, M., Smith, C., Shulman, J.A., y Steele, S. (2008). Universal Design of Instruction. Reflections of Students. En S. Burgstahler, Universal Design in Higher Education. From principles to practices (pp. 83-96). Cambridge, MA.: Harvard Education Press.

Education, Audiovisual and Culture Executive Agency. (2000). Key Data on Education in Europe (Key Data Series). Luxemburgo. Recuperado de: http://eacea.ec.europa. eu/education/eurydice/key_data_en.php.

Engleman, M., y Schmidt, M. (2007). Testing an experimental universally designed learning unit in a graduate level online teacher education course. Journal of Online Learning and Teaching, 3(2), 112-132.

European Agency for Development in Special Needs Education (2006). Educación Especial en Europa. Factores clave en la educación post-primaria (Monográfico). Bruselas, Bélgica. Recuperado de: https://goo.gl/3xPQRJ.

Horn, L., y Nevill, S. (2006). Profile of undergraduates in U.S. postsecondary education institutions: 2003-2004. Washington, DC.: National Center for Education Statistics, U.S. Department of Education.

Lewis, L., y Farris, E. (1999). An institutional perspective on students with disabilities in postsecondary education. Education Statistics Quartely, 1(3).

Mace, R. (1985). Universal Design, Barrier Free Environments for Everyone. Designers West.

Martínez, R., De Haro, R., y Escarbajal, A. (2010). Una aproximación a la educación inclusiva en España. Revista Educación Inclusiva, 3(1), 149-164.

McGuire, J., Scott, S., y Shaw, S. (2006). Universal Design and Its Aplications in Educational Environments. Remedial and Special Education, 27(3), 166-175.

Meo, G. (2008). Curriculum planning for all learners; applying universal design for learning (UDL) to a high school reading comprehension program. Preventing School Failure, 52(2), 21-30.

National Center for Universal Design for Learning (2012). UDL Guideline - Version 2.0. Recuperado de: http://www.udlcenter.org/aboutudl/udlguidelines.

Organización de las Naciones Unidas para la Educación, la Ciencia y la Cultura. (2008). Conferencia Internacional de Educación, 48a reunión: Informe final. Ginebra.

Pliner, S., y Johnson, J. (2004). Historical, theoretical, and foundational principles of universal instructional design in higher education. Equity \& Excellence in Education, 3, 105-113.

Roberts, K., Park, H., Brown, S., y Cook, B. (2011). Universal Design for Instruction in Postsecondary Education: A Systematic Review of Empirically Based Articles. Journal of Postsecondary Education and Disability, 24(1), 4-18. 
Rose, D., y Meyer, A. (2002). Teaching Every Student in the Digital Age: Universal Design for Learning. VA: ASCD.

Rose, D., Harbour, W., Johnston, C., Daley, S., y Abarbanell, L. (2006). Universal Design for Learning in Postsecondary Education: Reflections on Principles and their Application. Journal of Postsecondary Education and Disability, 19(2), 135-151.

Ruiz, R., Solé, L., Echeita, G., Sala, I., y Datsira, M. (2012). El principio del "Universal Design". Concepto y desarrollos en la enseñanza superior. Revista de Educación. doi:10-4438/1988-592X-RE-2010-359-100.

Sala, I., Autor, S., Giné, C., y Autor, E. (2014). Análisis de los distintos enfoques del paradigma del diseño universal aplicado a la educación. Revista Iberoamericana de Educación Inclusiva, 8(1), 143-152.

Sánchez, S., Díez, E., Verdugo, M.A., Iglesias, A., y Calvo, I. (2011). Atención a la diversidad en las titulaciones adaptadas al RD 1393/2007: Adaptación de una herramienta Web de autoevaluación curricular basada en los principios del diseño universal para el aprendizaje. En Jornadas de Innovación Docente en la Universidad de Salamanca (Universidad de Salamanca, España. Vicerrectorado de Docencia) (pp. 148-155). Salamanca. Recuperado de: http://gredos.usal.es/jspui/ handle/10366/112888.

Sánchez, S. (2015). Diseñando clases para todos desde las TIC. 25 aniversario Ceapat: 12 retos, 12 meses. Madrid: CEAPAT. Recuperado de: http://goo.gl/zCq2ae.

Schelly, C., Davies, P., y Spooner, C. (2011). Student perceptions of faculty implementation of universal design for learning. Journal of Postsecondary Education and Disability, 24(1), 17-30.

Spencer, A., y Romero, O. (2008). Engaging Higher Education Faculty in Universal Design. Addressing Needs to Students with Invisible Disabilities. En S. Burgstahler, Universal Design in Higher Education. From principles to practices (pp. 145-156). Cambridge, MA.: Harvard Education Press.

Spooner, F., Baker, J., Harris, A., Ahlgrim-Delzell, L., y Browder, D. (2007). Effects of Training in Universal Design for Learning on Lesson Plan Development. Remedial and Special Education, 28(2), 108-116.

Verdugo, M. A., y Rodríguez-Aguilella, A. (2012). La inclusión educativa en España desde la perspectiva de alumnos con discapacidad intelectual, de familias y de profesionales. Revista de Educación, 358, 450-470. doi: 10.4438/1988-592X-RE2011-358-086.

Yuval, L., Procter, E., Korabik, K., y Palmer, J. (2004). Evaluation Report on the Universal Instructional Design at the University of Guelph. Guelph: University of Guelph. Recuperado de: http://www.coles.uoguelph.ca/TSS/instructional_design/ highlights.aspx. 